\title{
EXTREME MONOPHONIC GRAPHS AND EXTREME GEODESIC GRAPHS
}

\author{
A. P. SANTHAKUMARAN AND P. TITUS
}

\begin{abstract}
For a connected graph $G=(V, E)$ of order at least two, a chord of a path $P$ is an edge joining two non-adjacent vertices of $P$. A path $P$ is called a monophonic path if it is chordless path. A monophonic set of $G$ is a set $S$ of vertices such that every vertex of $G$ lies on a monophonic path joining some pair of vertices in $S$. The monophonic number of $G$ is the minimum cardinality of its monophonic sets and is denoted by $m(G)$. A geodetic set of $G$ is a set $S$ of vertices such that every vertex of $G$ lies on a geodesic joining some pair of vertices in $S$. The geodetic number of $G$ is the minimum cardinality of its geodetic sets and is denoted by $g(G)$. The number of extreme vertices in $G$ is its extreme order $\operatorname{ex}(G)$. A graph $G$ is an extreme monophonic graph if $m(G)=\operatorname{ex}(G)$ and an extreme geodesic graph if $g(G)=\operatorname{ex}(G)$. Extreme monophonic graphs of order $p$ with monophonic number $p$ and $p-1$ are characterized. It is shown that every pair $a, b$ of integers with $0 \leq a \leq b$ is realized as the extreme order and monophonic number, respectively, of some graph. For positive integers $r, d$ and $k \geq 3$ with $r<d$, it is shown that there exists an extreme monophonic graph $G$ of monophonic radius $r$, monophonic diameter $d$, and monophonic number $k$. Also, we give a characterization result for a graph $G$ which is both extreme geodesic and extreme monophonic.
\end{abstract}

\section{Introduction}

By a graph $G=(V, E)$ we mean a finite undirected connected graph without loops or multiple edges. The order and size of $G$ are denoted by $p$ and $q$, respectively. For basic graph theoretic terminology we refer to Harary [4]. For vertices $x$ and $y$ in a connected graph $G$, the distance $d(x, y)$ is the length of a shortest $x$ - $y$ path in $G$. An $x-y$ path of length $d(x, y)$ is called an $x$-y geodesic. The neighborhood of a vertex $v$ is the set $N(v)$ consisting of all vertices $u$ which are adjacent with $v$. A vertex $v$ is an extreme vertex of $G$ if the subgraph induced by its neighbors is complete. An extreme vertex of $G$ is also called a simplicial vertex of $G$. The number of extreme vertices in $G$ is its extreme order ex $(G)$.

Received October 13, 2015, accepted October 11, 2016. 2010 Mathematics Subject Classification. 05C12.

Key words and phrases. Geodetic number, monophonic number, extreme order, extreme geodesic graph, extreme monophonic graph.

Corresponding author: P. Titus. 
A geodetic set of $G$ is a set $S$ of vertices such that every vertex of $G$ lies on a geodesic path joining some pair of vertices in $S$. The geodetic number of $G$ is the minimum cardinality of its geodetic sets and is denoted by $g(G)$. A geodetic set of cardinality $g(G)$ is called a $g$-set. The geodetic number of a graph was introduced in [1,5] and further studied in [2]. A graph $G$ is an extreme geodesic graph if $g(G)=\operatorname{ex}(G)$. Extreme geodesic graphs were introduced and studied in [4].

A chord of a path $P$ is an edge joining two non-adjacent vertices of $P$. A path $P$ is called a monophonic path if it is a chordless path. A set $S$ of vertices of $G$ is a monophonic set if each vertex $v$ of $G$ lies on an $x-y$ monophonic path for some $x, y \in S$. The minimum cardinality of a monophonic set of $G$ is the monophonic number of $G$ and is denoted by $m(G)$. The monophonic number of a graph was studied in [9].

For any two vertices $u$ and $v$ in a connected graph $G$, the monophonic distance $d_{m}(u, v)$ from $u$ to $v$ is defined as the length of a longest $u-v$ monophonic path in G. The monophonic eccentricity $e_{m}(\nu)$ of a vertex $v$ in $G$ is $e_{m}(v)=\max \left\{d_{m}(\nu, u): u \in V(G)\right\}$. The monophonic radius, $\operatorname{rad}_{m}(G)$ of $G$ is $\operatorname{rad}_{m}(G)=\min \left\{e_{m}(\nu): v \in V(G)\right\}$ and the monophonic diameter, $\operatorname{diam}_{m}(G)$ of $G$ is $\operatorname{diam}_{m}(G)=\max \left\{e_{m}(v): v \in V(G)\right\}$. A vertex $u$ in $G$ is a monophonic eccentric vertex of a vertex $v$ in $G$ if $e_{m}(u)=d_{m}(u, v)$. The monophonic distance was introduced in [7] and further studied in [8].

The following theorems will be used in the sequel.

Theorem 1.1 ([2]). Each extreme vertex of a graph $G$ belongs to every geodetic set of $G$.

Theorem $1.2([4])$. Let $G$ be a connected graph with at least three vertices. The following statements are equivalent:

(i) G is a block.

(ii) Every two vertices of G lie on a common cycle.

Theorem 1.3 ([9]). Each extreme vertex of a graph $G$ belongs to every monophonic set of $G$.

Theorem 1.4 ([9]). No cutvertex of a graph $G$ belongs to any minimum monophonic set of $G$.

Theorem 1.5 ([9]). Let $G$ be a connected graph with cutvertices and let $S$ be a monophonic set of $G$. If $x$ is a cutvertex of $G$, then every component of $G-x$ contains an element of $S$.

Theorem 1.6 ([5]). Let $G$ be a connected graph with cutvertices and let $S$ be a geodetic set of $G$. If $x$ is a cutvertex of $G$, then every component of $G-x$ contains an element of $S$. 


\section{Extreme monophonic graphs}

Definition 2.1. A graph $G$ is said to be an extreme monophonic graph if $m(G)=\operatorname{ex}(G)$.

For the graph $G$ given in Figure 2.1, $v_{1}$ and $v_{7}$ are the only two extreme vertices so that $\operatorname{ex}(G)=2$. The set $S=\left\{v_{1}, v_{7}\right\}$ is a minimum monophonic set of $G$ so that $m(G)=\operatorname{ex}(G)=2$. Therefore, $G$ is an extreme monophonic graph.

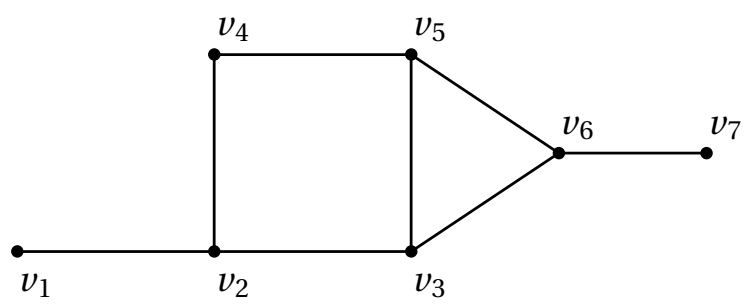

Figure 2.1: $G$.

For any nontrivial tree $T$ with $k$ end vertices, $\operatorname{ex}(T)=k$ and by Theorems 1.3 and 1.4, $m(T)=k$. Thus any nontrivial tree is an extreme monophonic graph. It is obvious that the cycle $C_{p}(p \geq 4)$ and the complete bipartite graph $K_{r, s}(2 \leq r \leq s)$ are not extreme monophonic graphs. By Theorem 1.3, we see that for any connected graph $G$ of order $p, 0 \leq \operatorname{ex}(G) \leq m(G) \leq$ $p$. It is an easy consequence of Theorem 1.3 that a connected graph $G$ of order $p \geq 2$ is an extreme monophonic graph with monophonic number $p$ if and only if $G=K_{p}$.

The following theorem gives a characterization of extreme monophonic graphs of order $p$ with monophonic number $p-1$.

Theorem 2.2. Let $G$ be a connected graph of order $p \geq 3$. Then $G$ is an extreme monophonic graph with $m(G)=p-1$ if and only if $G=K_{1}+\bigcup m_{j} K_{j}$, where $\sum m_{j} \geq 2$.

Proof. Let $G=K_{1}+\bigcup m_{j} K_{j}$, where $\sum m_{j} \geq 2$. Since $G$ has exactly one cutvertex and all other vertices are extreme, it follows from Theorems 1.3, 1.4 that $m(G)=p-1$. Conversely, let $G$ be an extreme monophonic graph with $m(G)=p-1$. Then there exists exactly one non-extreme vertex, say $x$, in $G$. If $p=3$, then $G$ is a path of order 3 and hence $G=K_{1}+\bigcup 2 K_{1}$. Let $p \geq 4$. We claim that $x$ is the cutvertex of $G$. Otherwise, $x$ lies on a smallest cycle, say $C$, of length at least 4. Then the neighbors of $x$ on $C$ do not belong to a minimum monophonic set of $G$ and hence $m(G) \leq p-2$, which is a contradiction. Hence $G-x$ has at least two components. We prove that each component is complete and $x$ is adjacent to every vertex of each component. Suppose there exists a component $B$, which is not complete. Let $u$ and $v$ be two vertices in $B$ such that $d(u, v) \geq 2$. Then by Theorem 1.2, both $u$ and $v$ lie on a common cycle and hence $u$ and $v$ lie on a smallest cycle of length at least 4 . Hence $m(G) \leq p-2$, which is a contradiction. 
Thus each component of $G-x$ is complete. Now, if $x$ is not adjacent to some vertex of a component, then $x$ lies on a monophonic path of length at least 3 so that $m(G) \leq p-2$, which is a contradiction. Hence $G=K_{1} \cup m_{j} K_{j}$, where $K_{1}$ is the vertex $x$ and $\sum m_{j} \geq 2$.

For any connected graph $G$, we have $0 \leq \operatorname{ex}(G) \leq m(G)$ and $2 \leq m(G) \leq p$. In view of this, we have the following realization result.

Theorem 2.3. For every pair $a, b$ of integers with $0 \leq a \leq b$ and $b \geq 2$, there exists a connected graph $G$ with $\operatorname{ex}(G)=a$ and $m(G)=b$.

Proof. We consider two cases, according to whether $a=0$ or $a \geq 1$.

Case (i) $a=0$. Let $F_{1}, F_{2}, \ldots, F_{i}, \ldots, F_{b}$ be such that each $F_{i}$ is a copy of $C_{4}$ with vertices $v_{i, 1}, v_{i, 2}$, $v_{i, 3}, v_{i, 4}$ for $i=1,2, \ldots, b$. Let $G$ be the graph obtained by identifying the vertices $v_{i, 1}$ of $F_{i}(1 \leq$ $i \leq b)$. The graph $G$ is shown in Figure 2.2. Since $b \geq 2$, $G$ has the unique cutvertex, say $x$.

Clearly, no vertex of $G$ is an extreme vertex and so $\operatorname{ex}(G)=0$. By Theorem 1.5, every monophonic set of $G$ contains at least one vertex from each component of $G-x$ and so $m(G) \geq$ $b$. Let $S=\left\{v_{1,3}, v_{2,3}, v_{3,3}, \ldots, v_{b, 3}\right\}$. Since $S$ is a monophonic set of $G$, it follows that $m(G)=|S|=$ b.

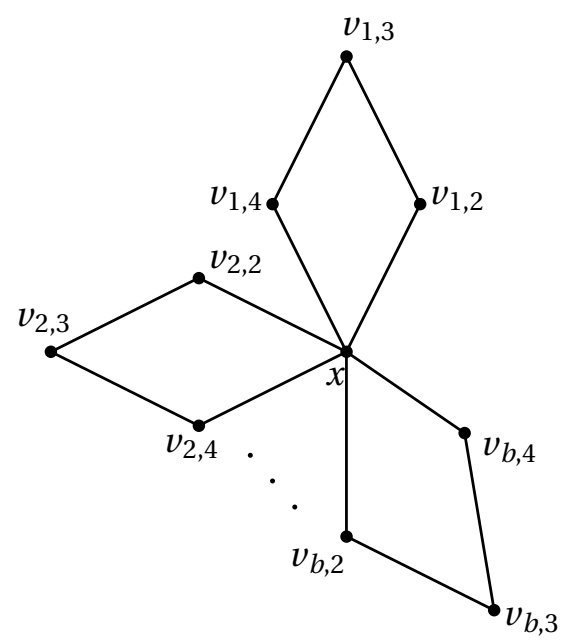

Figure 2.2: $G$

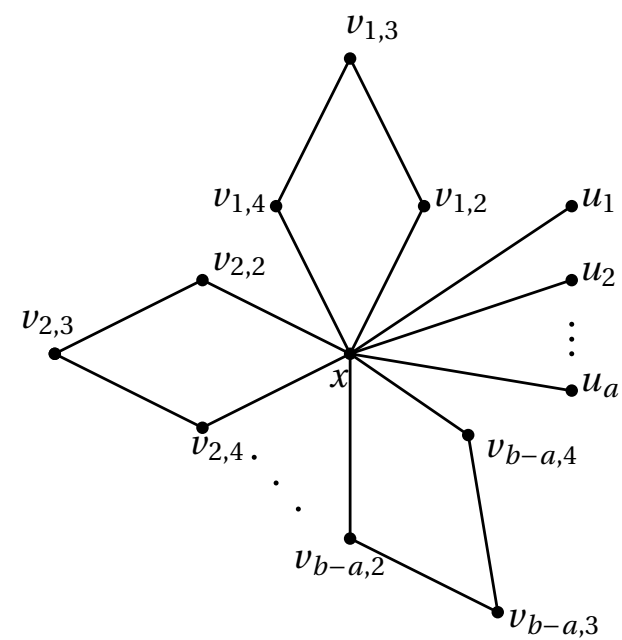

Figure 2.3: $G$

Case (ii) $a \geq 1$. If $a=b$, then the complete graph $G=K_{a}$ has the desired properties. If $a<b$, then we construct a graph $G$ as follows. Let $F_{1}, F_{2}, \ldots, F_{i}, \ldots, F_{b-a}$ be such that each $F_{i}$ is a copy of $C_{4}$ with vertices $v_{i, 1}, v_{i, 2}, v_{i, 3}, v_{i, 4}$ for $i=1,2, \ldots, b-a$. Let $K_{1, a}$ be the star at $x$ and let $U=\left\{u_{1}, u_{2}, \ldots, u_{a}\right\}$ be the set of endvertices of $K_{1, a}$. Let $G$ be the graph obtained by identifying the vertices $v_{i, 1}$ of $F_{i}(1 \leq i \leq b-a)$ and the vertex $x$ of $K_{1, a}$. The graph $G$ is shown in Figure 2.3 and it is clear that $\operatorname{ex}(G)=a$. 
By Theorems 1.3 and 1.5, every monophonic set of $G$ contains at least one vertex from each component of $G-x$ so that $m(G) \geq b$. Let $S=\left\{u_{1}, u_{2}, \ldots, u_{a}, v_{1,3}, v_{2,3}, \ldots, v_{b-a, 3}\right\}$. Since $S$ is a monophonic set of $G$, it follows that $m(G)=b$.

For any connected graph $G, \operatorname{rad}(G) \leq \operatorname{diam}(G) \leq 2 \operatorname{rad}(G)$. Ostrand [6] showed that every two positive integers $r$ and $d$ are realizable as the radius and diameter, respectively, of some connected graph. Ostrand's theorem can be extended to extreme monophonic graphs so that the monophonic number can also be prescribed.

Theorem 2.4. For positive integers $r, d$ and $k \geq 3$ with $r \leq d \leq 2 r$, there exists an extreme monophonic graph $G$ with $\operatorname{rad}(G)=r, \operatorname{diam}(G)=d$ and $m(G)=k$.

Proof. If $r=1$, then $d=1$ or 2. If $d=1$, then letting $G=K_{k}$, it follows from Theorem 1.3 that $m(G)=k$ and so $G$ is an extreme monophonic graph. Also, if $d=2$, then letting $G=K_{1, k}$, it follows from Theorem 1.3 that $m(G)=k$ and so $G$ is an extreme monophonic graph. Now, let $r \geq 2$. We construct a graph $G$ with the desired properties as follows:

Case 1. $r=d$. Let $n=k+2 r$. Let $a=\left\lceil\frac{k+4}{2}\right\rceil$ and $b=n-\left\lceil\frac{k}{2}\right\rceil$. Then it is clear that $2<a<b<n$. Let $C: x_{1}, x_{2}, \ldots, x_{a}, \ldots, x_{b}, \ldots, x_{n}, x_{1}$ be a cycle of order $n$.

Subcase (i). $k$ is even. Let $G$ be the graph obtained from $C$ by joining every pair of vertices of $\left\{x_{1}, x_{2}, \ldots, x_{a}\right\}$ and also every pair of vertices of $\left\{x_{b}, x_{b+1}, \ldots, x_{n}, x_{1}\right\}$. The graph $G$ is shown in Figure 2.4 for $k=6$ and $r=3$. Clearly, $S=\left\{x_{2}, x_{3}, \ldots, x_{a-1}, x_{b+1}, x_{b+2}, \ldots, x_{n}\right\}$ is the set of all extreme vertices of $G$ with $|S|=k$. It is easily verified that the eccentricity of each vertex of $G$ is $r$ so that $\operatorname{rad}(G)=\operatorname{diam}(G)=r$. It is clear that $S$ is the unique minimum monophonic set of $G$ so that $m(G)=k$ and $G$ is an extreme monophonic graph.

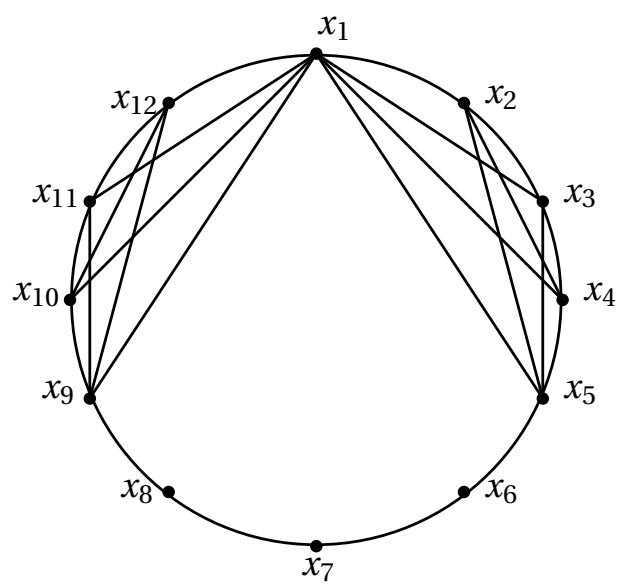

Figure 2.4: $G$ 
Subcase (ii). $k$ is odd. Let $G$ be the graph obtained from $C$ by joining every pair of vertices of $\left\{x_{2}, x_{3}, \ldots, x_{a}\right\}$, every pair of vertices of $\left\{x_{b}, x_{b+1}, \ldots, x_{n}\right\}$ and also adding the edge $x_{2} x_{n}$. The graph $G$ is shown in Figure 2.5 for $k=5$ and $r=3$. Then $S=\left\{x_{1}, x_{3}, x_{4}, \ldots, x_{a-1}, x_{b+1}, x_{b+2}, \ldots\right.$, $\left.x_{n-1}\right\}$ is the set of all extreme vertices of $G$ with $|S|=k$. It is clear that $1 \leq d\left(x_{1}, x_{i}\right) \leq r$ for $i=2,3, \ldots, n$ and $d\left(x_{1}, x_{\frac{n+1}{2}}\right)=d\left(x_{1}, x_{\frac{n+3}{2}}\right)=r$. Hence eccentricity of $x_{1}$ is $r$. Similarly, it can be verified that eccentricity of each of the remaining vertices is also $r$. Hence $\operatorname{rad}(G)=$ $\operatorname{diam}(G)=r$. It is clear that $S$ is the unique minimum monophonic set of $G$ so that $m(G)=k$ and $G$ is an extreme monophonic graph.

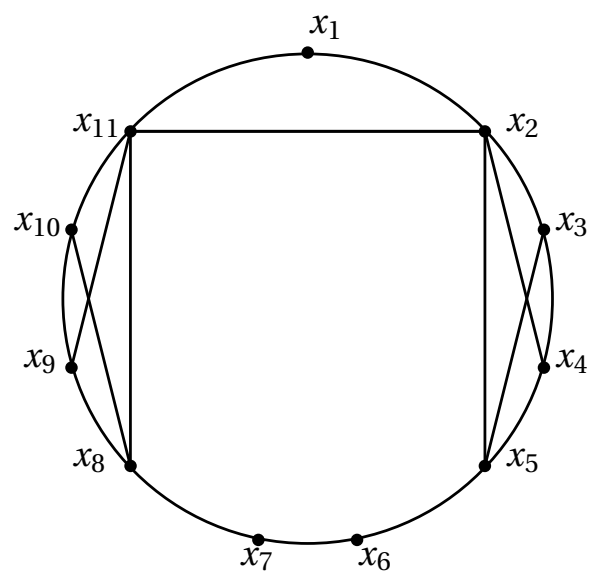

Figure 2.5: $G$

Case 2. $r<d \leq 2 r$. Let $P_{d+1}: u_{1}, u_{2}, \ldots, u_{r}, u_{r+1}, \ldots, u_{d+1}$ be a path of order $d+1$. Add $k-2$ new vertices $v_{1}, v_{2}, \ldots, v_{k-2}$ to $P_{d+1}$ and join each $v_{i}(1 \leq i \leq k-2)$ to $u_{d}$, there by producing the tree $G$ of Figure 2.6. Then $S=\left\{u_{1}, u_{d+1}, v_{1}, v_{2}, \ldots, v_{k-2}\right\}$ is the set of all extreme vertices of $G$ with $|S|=k$. It is clear that $e\left(u_{r+1}\right)=r, e\left(u_{1}\right)=d$ and $r \leq e(x) \leq d$ for all other vertices $x$ in $G$. Then $\operatorname{rad}(G)=r$ and $\operatorname{diam}(G)=d$. It is clear that $S$ is the unique minimum monophonic set of $G$ and so $m(G)=k$.
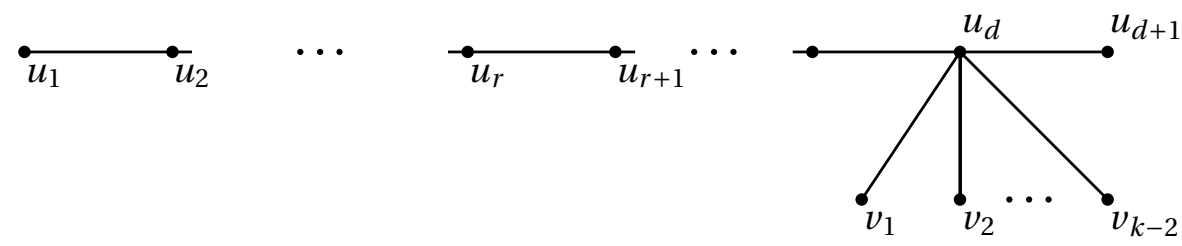

Figure 2.6: $G$ 
For any connected graph $G, \operatorname{rad}_{m}(G) \leq \operatorname{diam}_{m}(G)$. Santhakumaran and Titus [7] showed that every two positive integers $a$ and $b$ with $a \leq b$ are realizable as the monophonic radius and monophonic diameter, respectively, of some connected graph. This theorem can be extended to extreme monophonic graphs so that the monophonic number can also be prescribed when $\operatorname{rad}_{m}(G)<\operatorname{diam}_{m}(G)$.

Theorem 2.5. For positive integers $r, d$ and $k \geq 3$ with $r<d$, there exists an extreme monophonic graph $G$ with $\operatorname{rad}_{m}(G)=r, \operatorname{diam}_{m}(G)=d$ and $m(G)=k$.

Proof. We prove this theorem by considering two cases.

Case 1. $r=1$. Then $d \geq 2$. Let $C_{d+2}: v_{1}, v_{2}, \ldots, v_{d+2}, v_{1}$ be a cycle of order $d+2$. Let $G$ be the graph obtained by adding $k-2$ new vertices $u_{1}, u_{2}, \ldots, u_{k-2}$ to $C_{d+2}$ and joining each of the vertices $u_{1}, u_{2}, \ldots u_{k-2}, v_{3}, v_{4}, \ldots, v_{d+1}$ to the vertex $v_{1}$. The graph $G$ is shown in Figure 2.7. It is clear that $e_{m}\left(v_{1}\right)=1, e_{m}\left(v_{2}\right)=e_{m}\left(v_{d+2}\right)=d$ and $2 \leq e_{m}(x) \leq d-1$ for all other vertices $x$ in $G$. Hence $\operatorname{rad}_{m}(G)=1$ and $\operatorname{diam}_{m}(G)=d$. Let $S=\left\{u_{1}, u_{2}, \ldots, u_{k-2}, v_{2}, v_{d+2}\right\}$ be the set of all extreme vertices of $G$. By Theorem 1.3, every monophonic set of $G$ contains $S$. It is clear that $S$ is a monophonic set of $G$ and so $\operatorname{ex}(G)=m(G)=k$.

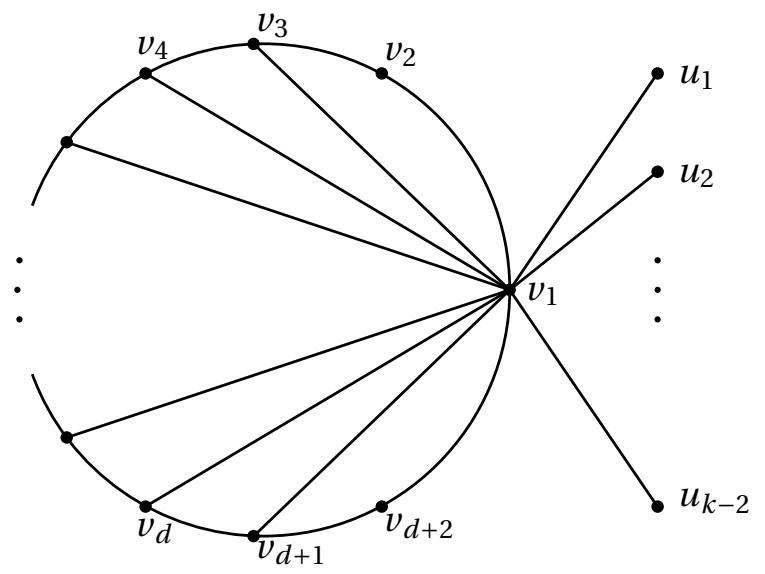

Figure 2.7: $G$ 


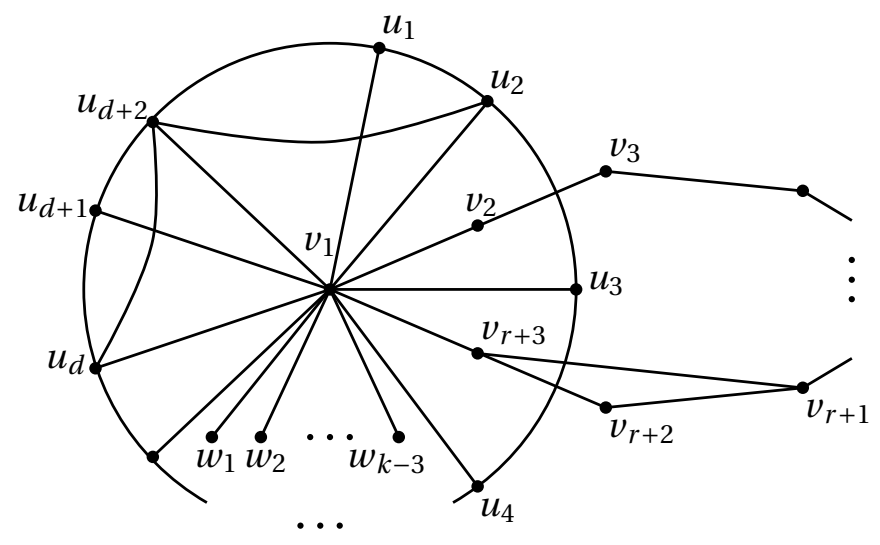

Figure 2.8: $G$

Case 2. $r \geq 2$. Let $C: v_{1}, v_{2}, \ldots, v_{r+3}, v_{1}$ be a cycle of order $r+3$ and let $W=K_{1}+C_{d+2}$ be a wheel with $V\left(C_{d+2}\right)=\left\{u_{1}, u_{2}, \ldots, u_{d+2}\right\}$ and let the vertex of $K_{1}$ be $v_{1}$. Let $H$ be the graph obtained from this by joining the vertices $v_{r+1}$ and $v_{r+3}$ of $C$, and also joining the vertex $u_{d+2}$ with the vertices $u_{2}$ and $u_{d}$ of $W$. Now, add $k-3$ new vertices $w_{1}, w_{2}, \ldots, w_{k-3}$ to the graph $H$ and join each vertex $w_{i}(1 \leq i \leq k-3)$ to the vertex $v_{1}$ and obtain the graph $G$ of Figure 2.8. It is easily verified that $r \leq e_{m}(x) \leq d$ for any vertex $x$ in $G$ and $e_{m}\left(v_{2}\right)=r, e_{m}\left(u_{1}\right)=d$. Then $\operatorname{rad}_{m}(G)=r$ and $\operatorname{diam}_{m}(G)=d$. Let $S=\left\{w_{1}, w_{2}, \ldots, w_{k-3}, v_{r+2}, u_{1}, u_{d+1}\right\}$ be the set of all extreme vertices of $G$. By Theorem 1.3, every monophonic set of $G$ contains $S$. It is clear that $S$ is a monophonic set of $G$ and so $\operatorname{ex}(G)=m(G)=k$.

Problem 2.6. For any three positive integers $r, d$ and $k \geq 3$ with $r=d$, does there exist an extreme monophonic graph $G$ with $\operatorname{rad}_{m}(G)=r$, $\operatorname{diam}_{m}(G)=d$ and $m(G)=k$ ?

Theorem 2.7. For any three positive integers $d, k$ and $p$ with $2 \leq d<p, 2 \leq k<p$ and $p-d-$ $k+2 \geq 0$, there exists an extreme monophonic graph $G$ of order $p$, monophonic diameter $d$ and $m(G)=k$.

Proof. Let $K_{p-d+1}$ be the complete graph with vertex set $\left\{w_{1}, w_{2}, \ldots, w_{p-d-k+2}\right.$, $\left.v_{1}, v_{2}, \ldots, v_{k-1}\right\}$. Now, add a new vertex $x$ to $K_{p-d+1}$ and let $H$ be the graph obtained from $K_{p-d+1}$ by joining $x$ with each $w_{i}(1 \leq i \leq p-d-k+2)$. Let $P: u_{0}, u_{1}, \ldots, u_{d-2}$ be a path of length $d-2$. Let $G$ be the graph obtained from $H$ and $P$ by identifying $u_{0}$ with $v_{1}$. Then $G$ has order $p$, monophonic diameter $d$ and the graph $G$ is shown in Figure 2.9. Let $S=$ $\left\{x, u_{d-2}, v_{2}, v_{3}, \ldots, v_{k-1}\right\}$ be the set of extreme vertices of $G$. It is clear that $S$ is a monophonic set of $G$ and so by Theorem $1.3, m(G)=\operatorname{ex}(G)=k$. Thus $G$ is an extreme monophonic graph with monophonic number $k$. 


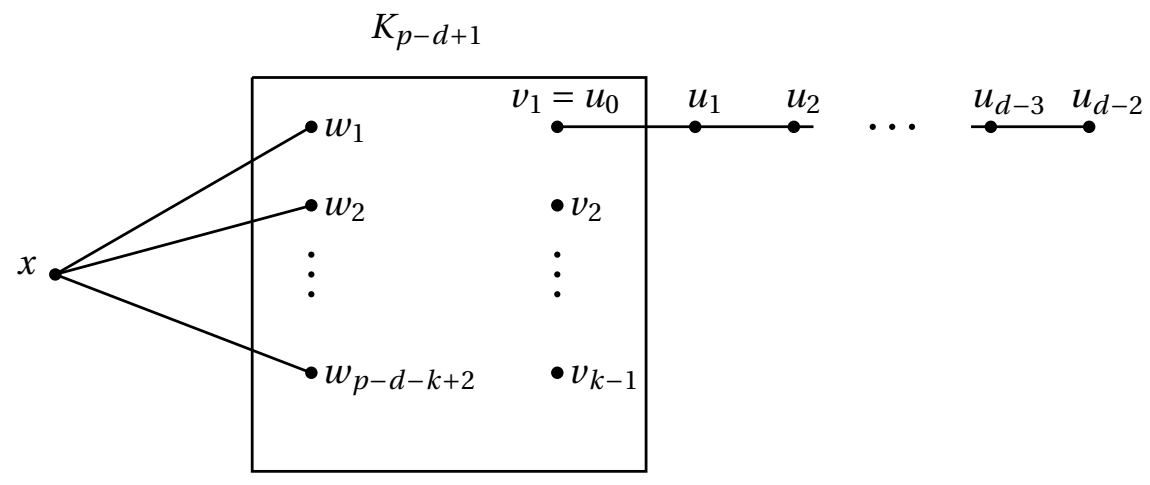

Figure 2.9: $G$

In the following theorem we construct a non-extreme monophonic graph $G$ of order $p$, monophonic diameter $\operatorname{diam}_{m}(G)=d$ and monophonic number $m(G)=k$ with data same as in Theorem 2.7 .

Theorem 2.8. For any three positive integers $d$, $k$ and $p$ with $2 \leq d<p, 2 \leq k<p$ and $p-d-k \geq$ 1 , there exists a non-extreme monophonic graph $G$ of order $p, \operatorname{diam}_{m}(G)=d$ and $m(G)=k$.

Proof. Let $W=K_{p-d-k}+C_{d+2}$ be a general wheel with $V\left(K_{p-d-k}\right)=\left\{v_{1}, v_{2}, \ldots, v_{p-d-k}\right\}$ and $V\left(C_{d+2}\right)=\left\{u_{1}, u_{2}, \ldots, u_{d+2}\right\}$. Now, add $k-2$ new vertices $w_{1}, w_{2}, \ldots, w_{k-2}$ to $W$ and join each vertex $w_{i}(1 \leq i \leq k-2)$ to every vertex of $K_{p-d-k}$. The graph $G$ is shown in Figure 2.10. It is easily verified that $1 \leq e_{m}(x) \leq d$ for any vertex $x$ in $G$ and $e_{m}\left(u_{1}\right)=d$. Then $\operatorname{diam}_{m}(G)=$ $d$. Let $S=\left\{w_{1}, w_{2}, \ldots, w_{k-2}\right\}$ be the set of all extreme vertices of $G$ and so $\operatorname{ex}(G)=k-2$. By Theorem 1.3, every monophonic set of $G$ contains $S$. It is clear that $S$ is not a monophonic set of $G$. Let $T=S \bigcup\left\{u_{2}, u_{d+2}\right\}$. It is clear that $T$ is a minimum monophonic set of $G$ and so $m(G)=$ $k$. Since $\operatorname{ex}(G)=k-2 \neq m(G), G$ is a non-extreme monophonic graph with $\operatorname{diam}_{m}(G)=d$ and $m(G)=k$.

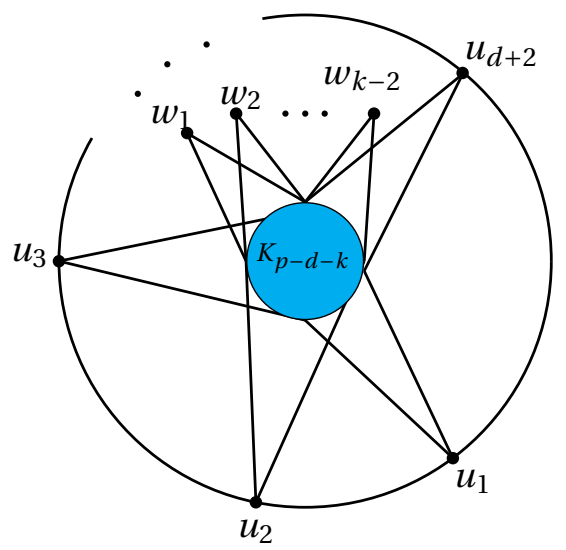

Figure 2.10: $G$ 


\section{Extreme monophonic graphs and extreme geodesic graphs}

Since every geodetic set is a monophonic set and every extreme vertex belongs to every geodetic set, it follows that every extreme geodesic graph is an extreme monophonic graph. However, the converse need not be true. For the graph $G$ given in Figure 2.1, $S=\left\{v_{1}, v_{7}\right\}$ is a minimum monophonic set so that $m(G)=\operatorname{ex}(G)=2$. Therefore, $G$ is an extreme monophonic graph. Since $S$ is not a geodetic set, $G$ is not an extreme geodesic graph. If $m(G)=g(G)=$ $\operatorname{ex}(G)$, then the graph $G$ is both extreme monophonic and extreme geodesic. We observe that if $G$ is extreme geodesic, then it is also extreme monophonic. In view of this observation, we have the following characterization theorem.

Theorem 3.1. A graph $G$ is both extreme geodesic and extreme monophonic if and only if every non-extreme vertex of $G$ lies on an $x-y$ geodesic for some extreme vertices $x$ and $y$ in $G$.

Proof. Let $S$ be the set of all extreme vertices of $G$. If every non-extreme vertex of $G$ lies on an $x-y$ geodesic for some extreme vertices $x$ and $y$ in $G$, then by Theorems 1.1 and 1.3, $S$ is both minimum geodetic set and minimum monophonic set of $G$. Hence $G$ is both extreme geodesic and extreme monophonic graph.

Conversely, let $G$ be both extreme geodesic and extreme monophonic graph. Then $\operatorname{ex}(G)=$ $g(G)=m(G)$. Since $\operatorname{ex}(G)=g(G), S$ is the unique minimum geodetic set. Hence every nonextreme vertex of $G$ lies on an $x-y$ geodesic for some vertices $x, y \in S$.

Theorem 3.2. For every pair $a, b$ of integers with $2 \leq a \leq b \leq 2 a$, there exists a connected graph $G$ which is neither extreme geodesic nor extreme monophonic such that $m(G)=$ a and $g(G)=b$.

Proof. For each integer $i$ with $1 \leq i \leq b-a$, let $C_{i}: v_{i, 0}, v_{i, 1}, v_{i, 2}, v_{i, 3}, v_{i, 4}, v_{i, 0}$ be a cycle of order 5 and for each integer $j$ with $1 \leq j \leq 2 a-b$, let $C_{j}^{\prime}: u_{j, 0}, u_{j, 1}, u_{j, 2}, u_{j, 3}, u_{j, 0}$ be a cycle of order 4 . Let $G$ be the graph obtained by identifying $v_{i, 0}$ of $C_{i}(1 \leq i \leq b-a)$ and $u_{j, 0}$ of $C_{j}^{\prime}(1 \leq j \leq 2 a-b)$. The graph $G$ is shown in Figure 3.1. It is clear that no vertex of $G$ is an extreme vertex of $G$. The set $M_{1}=\left\{u_{1,2}, u_{2,2}, \ldots, u_{2 a-b, 2}, v_{1,2}, v_{2,2}, \ldots, v_{b-a, 2}\right\}$ is a monophonic set of $G$ and so by Theorem 1.5, $m(G)=a$. Also, the set $S_{1}=\left\{u_{1,2}, u_{2,2}, \ldots, u_{2 a-b, 2}, v_{1,2}, v_{1,3}, v_{2,2}, v_{2,3}, \ldots, v_{b-a, 2}, v_{b-a, 3}\right\}$ is a geodetic set of $G$ and so by Theorem 1.6, $g(G)=b$. Hence $G$ is neither an extreme geodesic nor an extreme monophonic graph. 


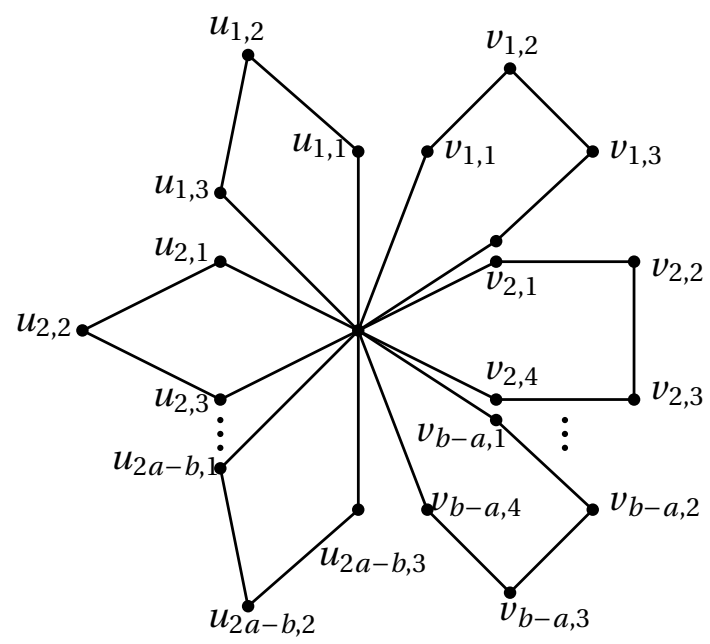

Figure 3.1: $G$

Problem 3.3. For every pair $a, b$ of integers with $2 \leq a \leq b$ and $b>2 a$, does there exist a connected graph $G$ which is neither extreme geodesic nor extreme monophonic such that $m(G)=a$ and $g(G)=b$ ?

Theorem 3.4. For every pair $a, b$ of integers with $2 \leq a<b \leq 2 a$, there exists a connected graph $G$ which is extreme monophonic but not extreme geodesic such that $m(G)=a$ and $g(G)=b$.

Proof. For each integer $i$ with $1 \leq i \leq b-a$, let $C_{i}: v_{i, 0}, v_{i, 1}, v_{i, 2}, v_{i, 3}, v_{i, 4}, v_{i, 0}$ be a cycle of order 5 and for each integer $j$ with $1 \leq j \leq 2 a-b$, let $C_{j}^{\prime}: u_{j, 0}, u_{j, 1}, u_{j, 2}, u_{j, 3}, u_{j, 0}$ be a cycle of order 4. Let $H$ be the graph obtained from $C_{i}$ and $C_{j}^{\prime}$ by identifying $v_{i, 0}$ of each $C_{i}(1 \leq i \leq b-a)$ and $u_{j, 0}$ of each $C_{j}^{\prime}(1 \leq j \leq 2 a-b)$. Now, let $G$ be the graph obtained from $H$ by (i) joining each $u_{j, 1}$ with $u_{j, 3}(1 \leq j \leq 2 a-b)$, and (ii) joining each $v_{i, 1}$ with $v_{i, 3}(1 \leq i \leq b-a)$. The graph $G$ is shown in Figure 3.2. Let $S=\left\{u_{1,2}, u_{2,2}, \ldots, u_{2 a-b, 2}, v_{1,2}, v_{2,2}, \ldots, v_{b-a, 2}\right\}$ be the set of all extreme vertices of $G$. Since $S$ is a monophonic set of $G$, it follows from Theorem 1.5 that $m(G)=a$. It is clear that $S$ is not a geodetic set of $G$ and $S_{1}=S \cup\left\{v_{1,3}, v_{2,3}, \ldots, v_{b-a, 3}\right\}$ is a geodetic set of $G$ and so by Theorem 1.6, $g(G)=b$. Hence $G$ is an extreme monophonic graph but it is not an extreme geodesic graph.

If $G$ is an extreme monophonic graph and it is not an extreme geodesic graph, then $m(G)<g(G)$. This leads to the following problem. 


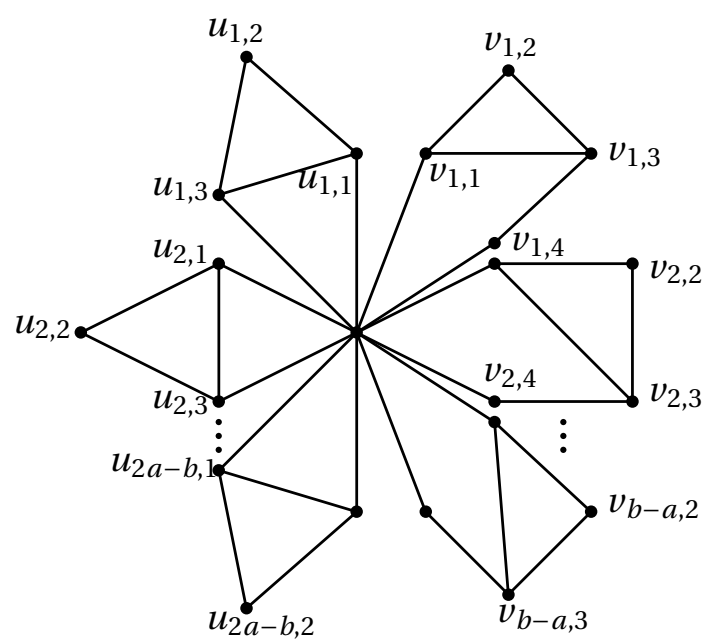

Figure 3.2: $G$

Problem 3.5. For every pair $a, b$ of integers with $2 \leq a<b$ and $b>2 a$, does there exist a connected graph $G$ which is extreme monophonic but not extreme geodesic such that $m(G)=a$ and $g(G)=b$ ?

\section{Acknowledgements}

The authors are thankful to the referee for the useful suggestions.

\section{References}

[1] F. Buckley and F. Harary, Distance in Graphs, Addison-Wesley, Redwood City, CA, 1990.

[2] G. Chartrand, F. Harary and P. Zhang, On the geodetic number of a graph, Networks, 39 (2002), 1-6.

[3] G. Chartrand and P. Zhang, Extreme Geodesic Graphs, Czechoslova Mathematical Journal, 52 (2002), 771-780.

[4] F. Harary, Graph Theory, Addison-Wesley, 1969.

[5] F. Harary, E. Loukakis and C. Tsouros, The geodetic number of a graph, Math. Comput. Modeling, 17 (1993), 87-95.

[6] P. A. Ostrand, Graphs with specified radius and diameter, Discrete Math., 4 (1973), 71-75.

[7] A. P. Santhakumaran and P. Titus, Monophonic Distance in Graphs, Discrete Mathematics, Algorithms and Applications, 3 (2011), 159-169.

[8] A. P. Santhakumaran and P. Titus, A note on monophonic distance in graphs, Discrete Mathematics, Algorithms and Applications, 4 (2012).

[9] A. P. Santhakumaran, P. Titus and K. Ganesamoorthy, On the monophonic number of a graph, J. Appl. Math. \& Informatics, 32 (2014), 255-266.

Department of Mathematics, Hindustan Institute of Technology and Science, Chennai - 603 103, India.

E-mail: apskumar1953@yahoo.co.in

Department of Mathematics, University College of Engineering Nagercoil, Anna University, Tirunelveli Region, Nagercoil - 629 004, India.

E-mail: titusvino@yahoo.com 